\title{
Hyperemesis gravidarum mit untypischer
} Ursache

\author{
Glioblastoma multiforme in der Schwangerschaft
}

\section{Fallbeschreibung}

Eine 28-jährige Patientin stellte sich im April 2017 erstmals in unserer Notfallklinik vor. Sie klagte über seit wenigen Monaten bestehende bitemporale, dumpf-drückende Kopfschmerzen, gelegentlich mit Übelkeit und Erbrechen, keine Photo- oder Phonophobie. Von den Neurologen wurde ein unauffälliger Status erhoben und die Diagnose eines Spannungskopfschmerzes gestellt. Sie wurde deshalb nach wenigen Stunden entlassen mit der Empfehlung, Amitriptylin $10 \mathrm{mg}$ zur Nacht einzunehmen und ambulant ein Schädel-MRT durchführen zu lassen. Dieses wurde nicht durchgeführt. Fünf Wochen später erfolgte eine Vorstellung in der gynäkologischen Ambulanz wegen Unterbauchschmerzen. Die Patientin befand sich jetzt in der 7. Schwangerschaftswoche (SSW). Anamnestisch ergaben sich folgende Informationen: IV. Gravida, Z. n. einem Spontanpartus, Z. n. 2 Frühaborten, chronische Zephalgien. Die gynäkologische Abklärung ergab eine regelrechte intrauterine Gravidität ohne Auffälligkeit, sodass noch am selben Tag eine Entlassung erfolgte.

Nach weiteren 3 Wochen (mittlerweile 10.SSW) stellte sie sich erneut wegen starker Übelkeit mit Erbrechen und moderatem Kopfschmerz vor. Es erfolgte deshalb die stationäre Aufnahme zur intravenösen Flüssigkeitssubstitution und Antiemese mit Dimenhydrinat i.v. Darunter kam es rasch zur Beschwerdebesserung, sodass sie nach wenigen Tagen mit einer antiemetischen Bedarfsmedikation wieder entlassen werden konnte. Nach 3 Tagen erfolgte eine erneute stationäre Aufnahme wegen Aggravation der Übelkeit und Fortbestehen der Kopfschmerzen. Trotz üblicher antiemetischer Therapie mit Dimenhydrinat und Flüssigkeitssubstitution zeigte sich keine Verbesserung. Die erneute neurologische Konsiluntersuchung ergab wiederum einen unauffälligen Status. Das nun durchgeführte Schädel-MRT zeigte eine ca. $5 \mathrm{~cm}$ durchmessende intrakra- nielle Raumforderung und einen Hydrocephalus occludens ( $\mathbf{A}$ bb. 1). Es erfolgte die rasche Verlegung in eine benachbarte neurochirurgische Klinik.

Hier erfolgte zunächst eine Shunteinlage mit Gewinnung einer Zytologie und Histologie. Diese ergaben den Befund eines Glioblastoma multiforme WHO-Grad IV im Bereich des Marklagers rechts mit thalamischer und dienzephaler Ausdehnung. Aufgrund der aggressiven, multimodalen Therapie wurde der Patientin ein Schwangerschaftsabbruch angeboten, der letztendlich in der 13.SSW vorgenommen wurde. Im Juli 2017 wurde der Tumor mikrochirurgisch subtotal reseziert. Die Patientin konnte danach ohne neurologische Defizite entlassen werden. Es folgten eine Radiochemotherapie (60 Gy) mit der Verwendung von Temozolomid und eine Tumorfeldtherapie, bei der über die Kopfhaut externe elektrische Wechselfelder appliziert werden.

Im Februar 2018 berichtete die Patientin über subjektives Wohlbefinden ohne Einschränkungen der Motorik. Eine VerlaufsMRT-Untersuchung zeigte jedoch eine leichte Progression des Tumors.

\section{Diskussion}

Übelkeit ist ein weit verbreitetes Problem in der Schwangerschaft. 50-90\% aller schwangeren Frauen sind davon betroffen. Als Zeichen schwangerschaftsbedingter hormoneller Veränderungen ist sie meist harmlos und sistiert mit zunehmendem Schwangerschaftsalter spontan [1].

Wenn die Übelkeit aber deutlich über das 1. Schwangerschaftsdrittel hinaus persistiert, an Intensität stark zunimmt und durch Medikamente in keiner Weise verbessert werden kann, ist eine weiterführende Diagnostik sinnvoll. An erster Stelle steht hier eine ausführliche Anamnese. Dabei gilt es die Dauer, den Charakter und alle möglichen Auslöser der Übelkeit

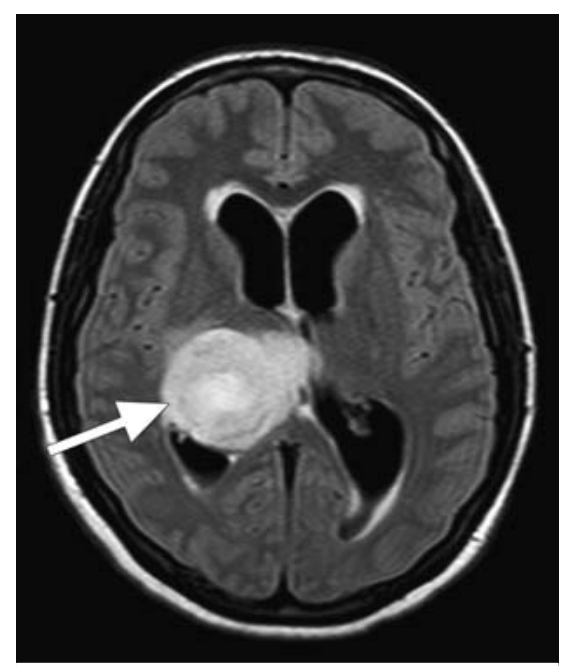

- Abb. 1 Natives Schädel-MRT einer Schwangeren mit Hyperemesis gravidarum und subchronischen Kopfschmerzen. Akuter obstruktiver Hydrozephalus bei hirneigenem, periventrikulärem Tumor (ca. $5 \times 4 \times 3,5 \mathrm{~cm}$ ) der Thalamusregion rechts. Quelle: Klinikum Memmingen

detailliert zu erfragen. Zusätzlich kann eine laborchemische Untersuchung Aufschlüsse über mögliche Ursachen der Übelkeit liefern.

Differenzialdiagnostisch kommen bei ausgeprägter Übelkeit in der Schwangerschaft verschiedene Erkrankungen infrage. Dazu zählen neben der Hyperemesis gravidarum als häufigste Ursache beispielsweise eine Gastroenteritis, die Präeklampsie, Migräne, Hyperthyreose, eine Lebensmittelintoleranz, Nierensteine, psychosozioökonomische Belastungen oder krankhafte Veränderungen im zentralen Nervensystem ( $\triangleright$ Tab. 1).

Klinische Warnzeichen für intrazerebrale Raumforderungen wie Blutungen oder Tumoren sind subchronische Kopfschmerzen, Übelkeit, Erbrechen, Wesensveränderungen sowie sensorische oder motorische Ausfallserscheinungen. In einem solchen Fall empfiehlt sich die rasche Durch- 
- Tab. 1 Differenzialdiagnosen der (Hyper-)Emesis gravidarum. gastrointestinale Ursachen

- Gastroenteritis

- Leber- und Gallenwegserkrankung

- Ileus/Subileus

- Pankreatitis/Appendizitis/Hepatitis

- Ulcus ventriculi oder duodeni

- Magenkarzinom

metabolische und toxische Ursachen

- diabetische Ketoazidose

- Morbus Addison

- Porphyrie

- Hyperthyreoidismus

- Vergiftung und Unverträglichkeit

- (Lebensmittel/Medikamente)

schwangerschaftsassoziierte Störungen

- (Hyper-)Emesis gravidarum

- schwangerschaftsinduzierte Cholestase

- Präeklampsie

- akute Leberverfettung
- Zwerchfellhernie urogenitale Ursachen

- Pyelonephritis

- Urämie

- Nephrolithiasis

- Prämenstruationssyndrom

- Endometriose

- Adnextorsion

- degenerative uterine Myome

zentralnervöse Ursachen

- Migräne

- vestibuläre Störungen

- Tumoren

- psychiatrische Ursachen
Tumorbildung, Blutung oder pathologischen Drucksteigerung im Bereich der Schädelhöhle sein.

- Das MRT-Schädel gilt als diagnostischer Goldstandard intrakranieller Raumforderungen.

\section{Interessenkonflikt}

Die Autoren geben an, dass kein Interessenkonflikt besteht.

\section{Autorinnen/Autoren}

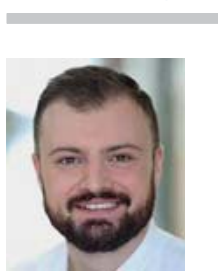

MUDr. Alex Horky

Gynäkologie und Geburtshilfe, Klinikum Memmingen

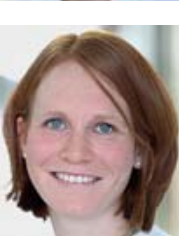

Dr. med. Kathrin Mühlen

Gynäkologie und Geburtshilfe, Klinikum Memmingen nauen Abklärung. Der vorliegende Fall unterstreicht die Notwendigkeit, dass bei Schwangeren mit therapieresistenter Hyperemesis, anhaltenden Kopfschmerzen und/oder zentralnervös bedingten klinischen Auffälligkeiten frühzeitig diese bildgebende Diagnostik zum Einsatz kommt.

Glioblastome (Glioblastoma multiforme, WHO-Grad IV) sind die häufigsten malignen Hirntumoren im Erwachsenenalter. Sie machen knapp 16\% aller hirneigenen Tumoren aus und treten bevorzugt bei Männern nach dem 50. Lebensjahr auf. Ihr rasches invasives Wachstum begünstigt peritumoröse Ödeme, wodurch es frühzeitig zu den oben genannten Beschwerden kommt. Nichtsdestotrotz sind Glioblastome bei Diagnosestellung meist schon so weit fortgeschritten, dass ihre komplette operative Entfernung ohne Funktionseinbußen für den Patienten nicht mehr möglich ist. Selbst nach einer subtotalen Resektion mit anschließender Bestrahlung und Chemotherapie ist das Fortschreiten der Erkrankung dauerhaft nicht zu verhindern. Abhängig vom Erkrankungsalter und der klinischen Beeinträchtigung zu Therapiebeginn lässt sich das Leben der Betroffenen durch die Therapie im Schnitt nur um 15-20 Monate verlängern [2].

\section{FAZIT FÜR DIE PRAXIS}

Am oben geschilderten Fall einer jungen schwangeren Patientin mit Glioblastom wird die klinische Relevanz neurologischer Differenzialdiagnosen bei der (Hyper-)Emesis gravidarum deutlich. Zum raschen Ausschluss einer zentralnervösen Pathologie sollten bei anhaltender Übelkeit, chronischen Kopfschmerzen, neurologischen Symptomen oder psychischen Auffälligkeiten eine neurologische Mitbetreuung und eine entsprechende Bildgebung in Form eines MRT des Schädels erfolgen.

- Übelkeit in der Schwangerschaft ist in der Regel harmlos und selbst limitierend.

- Bei anhaltender, therapieresistenter Übelkeit ist eine weiterführende, interdisziplinäre Abklärung sinnvoll.

- (Sub-)chronische Kopfschmerzen, neurologische Symptome und neuropsychologische Auffälligkeiten in Form von Wesensveränderungen oder Verhaltensstörungen können Zeichen einer

\section{KERNAUSSAGEN}

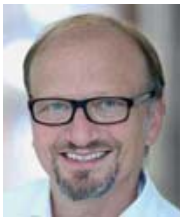

PD Dr. med. Felix Flock

Gynäkologie und Geburtshilfe, Klinikum Memmingen

Korrespondenzadresse

PD Dr. med. Felix Flock

Klinik für Frauenheilkunde und Geburtshilfe Klinikum Memmingen

Bismarckstraße 23

87700 Memmingen

Felix.Flock@klinikum-memmingen.de

Literatur

[1] Mylonas I, Gingelmaier A, Kainer F et al. Erbrechen in der Schwangerschaft. Dt Arztebl 2007; 104: A-1821/B-1604/C-1544

[2] Goldbrunner R, Ruge M, Kocher $M$ et al. Behandlung von Gliomen im Erwachsenenalter. Dt Arztebl Int 2018; 115: 356-364

\section{Bibliografie}

DOI https://doi.org/10.1055/a-0725-9857 Geburtsh Frauenheilk 2019; 79: 254-255 @ Georg Thieme Verlag KG Stuttgart · New York I ISSN 0016-5751 